\title{
ESTUDO DA HIPERTENSÃO ARTERIAL SISTÊMICA: REPERCUSSÕES QUANTO A ADESÃO AO TRATAMENTO
}

\author{
A STUDY ON HYPERTENSION: EFFECTS OF ADHESION TO TREATMENT
}

\author{
Thiago Rodrigues Pinto de Oliveira ${ }^{1}$, Leila Aparecida Kauchakje Pedrosa ${ }^{2}$, Rejane \\ Maria Dias de Abreu Gonçalves ${ }^{3}$
}

\begin{abstract}
Resumo: A Hipertensão Arterial Sistêmica (HAS), quando não tratada, constitui um dos principais fatores de risco para o aparecimento de doenças cardiovasculares (doença cerebrovascular, doença arterial coronariana, insuficiência cardíaca, insuficiência renal crônica e doença vascular de extremidade). O estudo tem os seguintes objetivos: identificar, na literatura, os fatores de risco sócio-econômicos e epidemiológicos que interferem no desenvolvimento da doença, os índices de adesão e os métodos (diretos e indiretos) que avaliam a aderência à prescrição médica; descrever o impacto na não adesão ao tratamento e fatores (relacionados ao paciente, à terapêutica e ao sistema de saúde) que influenciam na adesão. As bases acessadas foram LILACS, BDENF, SCIELO, Portal da CAPES e a V Diretriz Brasileira de Hipertensão Arterial (2006). A busca sistematizada foi realizada por meio dos Descritores em Ciências da Saúde (DECS) "Hipertensão Arterial Sistêmica"; adesão ao tratamento" e "fatores de risco", recuperando, apenas, os artigos publicados em periódicos disponíveis nessas bases de dados, entre os anos de 1998 a 2008 . Foram encontrados 98 artigos, sendo selecionados 11 que atendiam aos critérios definidos no enfoque do estudo da hipertensão arterial e a adesão ao tratamento. A análise das publicações demonstrou que a HAS é uma doença que tem tratamento eficaz, no entanto, continua a gerar altas taxas de morbimortalidade pela falta de adesão e/ou tratamento inadequado. O hipertenso necessita de um tratamento correto, condizente com sua situação social, econômica e cultural, bem como o acesso à medicação associada ao seu estilo de vida para atingir o nível pressórico adequado. É uma doença que precisa ser compreendida pela família, pelo paciente e pela equipe de saúde para melhor controle da pressão arterial e prevenção das complicações.
\end{abstract}

Palavras-chave: Hipertensão Arterial Sistêmica. Adesão ao tratamento. Fatores de risco

Abstratct: High blood pressure (HBP), if not treated, is a major risk factor for the emergence of cardiovascular disease (cerebral-vascular disease, coronary artery disease, heart failure, chronic renal failure and peripheral vascular disease). This study has the following goals: identify in the literature socio-economic and epidemiological risk factors for developing diseases, rates of adhesion and methods (direct and indirect) to assess adhesion to prescription; describe the impact on non-adhesion to treatment and factors (related to the patient, therapy and the health care system) that influence it. We accessed LILACS, BDENF, SCIELO, Portal of CAPES and V Brazilian Guidelines on Hypertension (2006) databases. A systematic search was performed using the Descriptors in Health Sciences (DECS) "hypertension" with treatment "and" "risk factors", retrieving only articles published in journals available in the databases from 1998 to 2008. We found 98 items, and selected 11 that met the criteria focus on the study. An analysis of publications showed that HBP is a disease that

\footnotetext{
${ }^{11}$ Aluno do $4^{\circ}$ ano do Curso de Enfermagem da Universidade Federal do Triângulo Mineiro (UFTM). Telefone: (34) 3332-1126

${ }^{22}$ Professora Doutora Adjunto da Escola de Enfermagem da UFTM. e-mail:

leila.kauchakje@terra.com.br

3 Enfermeira discente do Curso de Pós-graduação Mestrado Stricto Sensu Atenção à Saúde da

UFTM. e-mail: rejane.abreu@bol.com.br
} 
has effective treatment but continues to have high rates of morbidity and mortality due to lack of adhesion and / or inadequate treatment. Hypertension treatment must be correct, consistent with the patients' social, economic and cultural situation, as well as to availability of medication related to their lifestyle to achieve the appropriate pressure level. It is a disease that needs understanding by the family, the patient and the health team for controling blood pressure and thus prevent complications.

Key words: Hypertension. Adhesion. Risk factors

\section{Introdução}

A Pressão Arterial (PA) é definida como sendo o produto da relação entre o Débito Cardíaco (DC) e a Resistência Periférica (RP). O DC é igual ao produto entre Freqüência Cardíaca (FC) e o Volume Sistólico (VS). Temos, então, PA = DC x RP ou $P A=F C \times V S \times R P$. A pressão é exercida pelo fluxo sangüíneo através do coração e dos vasos sangüíneos na circulação normal. Alterações no DC e/ou na RP podem elevar a PA a valores anormais, com Pressão Arterial Sistólica (PAS) maior que $140 \mathrm{mmHg}$ e Pressão Arterial Diastólica (PAD) superior a $90 \mathrm{mmHg}$, durante um intervalo de tempo sustentado, na média de duas ou mais aferições da PA, em dois ou mais contatos com o profissional de saúde, sendo assim, definida a Hipertensão Arterial Sistêmica - HAS ${ }^{(1)}$.

A classificação da HAS, em pessoas com mais de 18 anos, é a seguinte: estágio $1 \mathrm{com}$ valores entre $140 \mathrm{mmHg}$ a $159 \mathrm{mmHg}$ para a PAS e entre $90 \mathrm{mmHg}$ a $99 \mathrm{mmHg}$ para PAD; estágio 2 entre $160 \mathrm{mmHg}$ a $179 \mathrm{mmHg}$ para PAS e entre 100 $\mathrm{mmHg}$ a $109 \mathrm{mmHg}$ para PAD; estágio 3 com PAS maior ou igual a $180 \mathrm{mmHg}$ e PAD maior ou igual a 110 e a Hipertensão sistólica isolada em que a PAS é maior ou igual a $140 \mathrm{mmHg}$ e a PAD é menor que $90 \mathrm{mmHg}$. Essa classificação revela a relação direta entre o risco de morbidade e mortalidade da HAS, mostrando que, quanto maior for a PAS ou PAD, maior será o risco ${ }^{(2)}$.

Em 2003, no Brasil, 27,4\% das mortes ocorreram por doenças cardiovasculares (cerebrovascular, doença arterial coronariana, insuficiência cardíaca, insuficiência renal crônica e doença vascular de extremidade) relacionadas, diretamente, com a HAS. Os óbitos atingiram 37\% quando excluídas mortes por causas mal definidas e violência. O maior número de mortes, no Brasil, em todas as regiões, é o Acidente Vascular Cerebral (AVC), com maior prevalência no sexo feminino. A HAS e as doenças relacionadas com a PA apresentam um alto número de internações, sendo que a insuficiência cardíaca é a causa mais comum 
de hospitalizações entre as doenças cardiovasculares, apresentando elevados custos médicos e socioeconômicos ${ }^{(2)}$.

Com o intuito de reduzir a morbidade e a mortalidade, o tratamento proposto pela equipe de saúde é de fundamental importância, que apresenta um plano de controle barato e simples: modificações no estilo de vida (perda de peso, limitação da ingesta de álcool, aumento da atividade física, redução da ingesta de sódio, não fumar); dar explicações claras, ao paciente, sobre a doença; fazer prescrições medicamentosas de forma mais simplificada possível; facilitar a aquisição do medicamento pelo paciente e a escolha da medicação certa ${ }^{(1-3)}$.

A terapia anti-hipertensiva é extremamente eficaz em reduzir a Pressão Arterial (PA). Contudo, o percentual de pacientes que alcançam os alvosterapêuticos é baixo. A não adesão ao tratamento é um dos mais importantes problemas enfrentados, pois gera custos substanciais pelas baixas taxas de controle alcançadas em todo o mundo, o que acaba aumentando a morbimortalidade decorrente dessa síndrome ${ }^{(4)}$.

No mundo e no Brasil, são poucos os estudos que descrevem índices de adesão entre os pacientes hipertensos. Os estudos, muitas vezes, não são comparáveis por abordarem diferentes perfis de indivíduos e usarem métodos diferentes para identificar a adesão. A adesão terapêutica, nos últimos anos, tornouse um grande problema, enfrentado na prática médica pela sua complexidade. Cerca de $40 \%$ a $60 \%$ dos pacientes não fazem uso da medicação prescrita. E quando a falta de adesão relaciona-se a itens como estilo de vida (dieta, sedentarismo, tabagismo, etilismo, entre outros fatores) a porcentagem aumenta ainda mais. Apesar de haver limitações dos dados disponíveis, os índices de adesão da HAS ficam abaixo da recomendação de $80 \%$ em diversos países. Em mais de dois terços de hipertensos, a PA é descontrolada pela falta de adesão ao tratamento(6).

Adesão ao tratamento, medicamentoso ou não, é fundamental para o sucesso da terapia instituída pela equipe de saúde. O termo adesão vai muito além do simples seguimento da prescrição medicamentosa, e envolve aspectos que influenciam a adesão ao tratamento, relacionados ao paciente (sexo, idade, etnia, estado civil, escolaridade e nível socioeconômico); à doença (cronicidade, ausência de sintomas e conseqüências tardias); às crenças de saúde, hábitos de vida e culturais (percepção da seriedade do problema, desconhecimento, experiência com 
a doença no contexto familiar e auto-estima); ao tratamento dentro do qual existe a qualidade de vida (custos, efeitos indesejáveis, esquemas terapêuticos complexos); à instituição política de saúde (acesso ao serviço de saúde, tempo de espera versus tempo de atendimento); e, finalmente, ao relacionamento com a equipe de saúde ${ }^{(5)}$.

\section{Objetivos}

Identificar, na literatura, os fatores de risco para desenvolver a HAS, verificar os conceitos, os índices de adesão ao tratamento e os métodos que avaliam a adesão, com o intuito de descrever o impacto social na não adesão à terapêutica.

\section{Metodologia}

Estudo bibliográfico, descritivo e de revisões sistemáticas na literatura. Para o alcance do objetivo deste estudo, foi realizado levantamento bibliográfico na biblioteca virtual em saúde BIREME, na base de dados LILACS (Literatura LatinoAmericana e do Caribe/Biblioteca Virtual em Saúde - Bireme), SCIELO (SCIENTIF ELETRONIC LIBRARY ONLINE) e no portal de periódicos da CAPES. Foram utilizadas as palavras chave "Hipertensão Arterial Sistêmica" "adesão ao tratamento" e "fatores de risco", recuperando, apenas, os artigos publicados em periódicos disponíveis nessas bases de dados entre os anos de 1998 a 2008 . Foram encontradas 98 publicações a partir da leitura exploratória do material nas bases de dados. Definiu-se a exclusão das referências que não atendiam ao propósito do estudo, sendo utilizados apenas 11 artigos.

Utilizaram-se referências da literatura tradicional e a $V$ Diretriz Brasileira de Hipertensão Arterial (2006).

As publicações foram estudadas, sendo os artigos foram lidos na íntegra e analisados de acordo com as seguintes dimensões: identificação dos fatores de risco da HAS, conceitos, índices e métodos de avaliação da adesão; impacto social da não adesão e fatores que influenciam na adesão ao tratamento.

\section{Resultados e Discussões}


O material de estudo constituiu-se de 11 referências publicadas entre 2002 e 2008. Com base nos títulos dos artigos, verificou-se que a temática mais abordada no levantamento bibliográfico foi a adesão ao tratamento anti-hipertensivo, o que foi citado em nove publicações (81,81\%), sendo estudados aspectos como conceito de adesão, dificuldades de aderência ao tratamento, índices de adesão, impacto social da HAS não tratada, e fatores que influenciam na adesão. Nas duas publicações restantes $(18,18 \%)$, foram extraídos conceitos, epidemiologia e fatores de risco da HAS. Para a escolha das publicações, os critérios de inclusão pré-estabelecidos foram respeitados.

Com relação à primeira dimensão de análise, verificou-se, nos dados levantados, que a idade, sexo, etnia, fatores sócio-econômicos, o excesso de sal, a obesidade, o uso de bebidas alcoólicas e o sedentarismo são os fatores de risco mais prevalentes para o desenvolvimento da $\mathrm{HAS}^{(2)}$.

Os estudos demonstraram que a pressão arterial aumenta linearmente com o avanço da idade. Em pessoas jovens, a HAS ocorre mais freqüentemente com a elevação exclusiva da PAD. A partir dos 60 anos, o principal componente é a PAS. Quanto ao sexo e etnia, estimativas globais sugerem taxas de hipertensão mais elevadas para indivíduos do sexo masculino até os 50 anos e para o sexo feminino, acima de 60 anos. Mulheres afrodescendentes apresentam excesso de risco cardiovascular de, até, $130 \%$ em relação às mulheres brancas ${ }^{(6)}$.

Em relação ao desenvolvimento sócio-econômico, os dados evidenciaram que a relação está inversamente proporcional, ou seja, quanto menor o nível socioeconômico, maior é a prevalência de HAS e de fatores de risco para elevar a PA, além de apresentar maior risco de lesão de órgão alvo e eventos cardiovasculares.

Hábitos alimentares, ingestão de sal e álcool, Índice de Massa Corporal (IMC) aumentado, estresse psicossocial, menor acesso aos cuidados de saúde e nível educacional são fatores associados. O excesso de sal na dieta está diretamente relacionado à HAS. Aumento da PA e avanço da idade estão diretamente associados. Essa relação é maior em populações com alta ingestão de sal. Povos que ingerem baixa quantidade de sal têm menor prevalência de HAS e a PA não se eleva com a idade. Entre os índios da tribo Yanomami, que apresenta baixa ingestão 
de sal, não foram observados casos de HAS. Na população urbana brasileira, indivíduos com nível sócioeconômico menor, apresentam maior ingestão de sal ${ }^{(2)}$.

De acordo com os dados, a obesidade está relacionada com o total de $20 \%$ a $30 \%$ dos casos de HAS. É responsável por excesso de massa corporal, sendo que $75 \%$ dos homens e $65 \%$ das mulheres apresentam hipertensão diretamente associada a sobrepeso e obesidade. Ganho de peso se relaciona fortemente com o aumento da PA. No entanto, nem todos os obesos tornam-se hipertensos. Foi observado, através de estudos, que o ganho de peso e aumento da circunferência da cintura são prognósticos importantes da HAS e a obesidade central aumenta o risco de doenças cardiovasculares. A obesidade central está mais associada com níveis de PA do que adiposidade total. A redução de peso acarreta redução da $\mathrm{PA}^{(4)}$.

Atualmente, tem aumentado a prevalência das pessoas em relação ao consumo de bebidas alcoólicas como cerveja, vinho e destilados. Consequentemente, acontece o aumento da pressão arterial. $O$ efeito varia com o gênero e a magnitude está associada à quantidade de etanol e a freqüência de consumo. Consumo leve a moderado de álcool não está definitivamente estabelecido. Existe uma redução média de $3,3 \mathrm{mmHg}(2,5 \mathrm{mmHg}$ a $4,1 \mathrm{mmHg})$ na PAS e $2 \mathrm{mmHg}(1,5 \mathrm{mmHg}$ a 2,6 $\mathrm{mmHg})$ na PAD, com a redução de ingestão de etanol. O consumo de bebidas alcoólicas fora de refeições aumenta o risco da hipertensão, independentemente da quantidade de álcool ingerida(2).

Pessoas sedentárias apresentam risco $30 \%$ maior de desenvolver a HAS que os ativos. O efeito hipotensor do exercício aeróbio é maior em indivíduos hipertensos que normotensos. Já o exercício resistido possui efeito hipotensor semelhante, mas menos consistente.

Sobre a segunda dimensão, os artigos apreendidos são todos oriundos de trabalhos originais de pesquisa, sendo esse dado considerado relevante. Não é de se surpreender que haja poucos trabalhos de revisão de literatura, pois, se o campo ainda está sendo construído, o esperado é que primeiro tenha uma relativa produção para que, posteriormente, seja feita apreciação do tipo e qualidade dessa produção, até para nortear futuras pesquisas.

O Projeto de Adesão da Organização Mundial de Saúde (1979) tem como definição de adesão a tratamentos crônicos uma junção de duas definições de Haynes (1979), Rand (1993), que conceituam adesão como o grau em que o comportamento de uma pessoa, representado pela ingestão de medicação, 
mudanças no estilo de vida, dieta adequada ao hipertenso e seguimento das recomendações dos profissionais de saúde. Para Miller et al. (1997), adesão ao tratamento é o meio para se alcançar um fim, abordando saúde para manutenção ou melhora de sinais e sintomas da doença. Car et al. (1991) definem adesão como sendo o grau de cumprimento das medidas terapêuticas indicadas, sejam elas medicamentosas ou não, para a manutenção da PA em níveis normais. Definição de adesão deve sempre abranger a vontade do indivíduo em participar e colaborar no tratamento, o que não é abordado em algumas concepções ${ }^{(7)}$.

A adesão é freqüentemente descrita como variável dicotômica (adesão versus não adesão), podendo variar de zero a mais de $100 \%$ em pacientes que usam mais do que as medicações prescritas pelo médico, tornando difícil detectar a falta de adesão e, mais ainda, quantificá-la. Não existe um consenso acerca do padrão que constitui a taxa de adesão adequada no tratamento da HAS. Alguns estudos consideram taxas acima de $80 \%$ aceitáveis. No mundo e no Brasil, poucos estudos descrevem índices de adesão entre os pacientes hipertensos. Os estudos, muitas vezes, não são comparáveis, por abordarem diferentes perfis de indivíduos e usarem métodos diferentes para identificar a adesão ${ }^{(8)}$.

A adesão terapêutica, nos últimos anos, tornou-se um grande problema enfrentado, na prática médica, pela sua complexidade. Cerca de $40 \%$ a $60 \%$ dos pacientes não fazem uso da medicação prescrita. E, quando a falta de adesão relaciona-se a itens como estilo de vida (dieta, sedentarismo, tabagismo, etilismo, entre outros fatores) a porcentagem aumenta. Apesar de haver limitações dos dados disponíveis, os índices de adesão da HAS ficam abaixo da recomendação de $80 \%$ em diversos países, mais de dois terços de hipertensos. A PA é descontrolada pela falta de adesão ao tratamento ${ }^{(6,7,8)}$.

Os métodos de avaliação da aderência ao tratamento consistem em métodos diretos e indiretos. Os métodos indiretos são os mais comumente usados, pois são de fácil utilização e não geram custos para o sistema de saúde e para o paciente, e permitindo, ainda, uma melhor interação entre o paciente e a equipe de saúde na avaliação da sua adesão ao tratamento.

Os métodos de avaliação a adesão estão descritos de acordo com os métodos indiretos e métodos diretos ${ }^{(9)}$.

Os métodos indiretos são abordados na literatura como um método simples, fácil de ser obtido e com alta especificidade, voltados para o paciente, como o 
relatório diário do tratamento e a contagem de comprimidos que requerem a participação do paciente. Na utilização de $80 \%$ ou mais dos comprimidos, é considerado um bom aderente ao tratamento. A relação entre controle de PA e número de pílulas tomadas é um bom marcador de adesão. Um dos poucos estudos que analisou essa relação mostrou que, dos pacientes com adesão maior que $80 \%$, apenas $32 \%$ estavam com a PA controlada e $24 \%$ sem controle; no grupo com adesão menor que $80 \%, 16 \%$ estavam controlados e $28 \%$, sem controle. O indivíduo que apresentou uma boa adesão ao tratamento, avaliada pela contagem de pílula, não quer dizer que ele fez o uso das pílulas. Alguns pacientes podem ter retirado comprimidos do frasco e oferecido a outro hipertenso ou mesmo tê-los jogado fora ${ }^{(9)}$.

Outros métodos que não dependem apenas do sujeito, mas, do atendimento do médico, em resposta ao tratamento, como a opinião do profissional, o reabastecimento de receitas, a resposta clínica e a monitoração. Esses fatores podem interferir na adesão ao tratamento. Às vezes, o mau controle pressórico é interpretado como não aderência ao tratamento, sendo, muitas vezes, simplista, visto que outras razões para o mau controle da pressão poderão estar presentes e não lembradas pelo médico.

O reabastecimento de receitas é um método que pode oferecer a média de adesão ao tratamento. No entanto, é uma forma mais trabalhosa, que necessita de um programa para computador, além da escolha de um grupo de farmácias, nas quais as informações colhidas possam apresentar credibilidade. É um método sujeito aos vícios de amostragem.

A resposta clínica é um método mais utilizado por ser simples e fácil e que parte do princípio de que o controle da PA é uma variável direta e única de adesão ao tratamento, o que, reconhecidamente, não é verdade, pois outros fatores estão envolvidos no controle adequado da PA.

Quanto aos métodos diretos, os estudos descrevem dois tipos: a análise biológica do medicamento e a adição de um traçador no organismo. O primeiro realiza a dosagem pelo sangue ou na urina de um de seus metabólitos. É considerado método padrão ouro para avaliar a adesão. No entanto, apresenta alguns inconvenientes, tornando sua execução impraticável. Essa análise requer metodologia sofisticada e onerosa, pode sofrer alterações de outros fatores biológicos, como por exemplo: proporção absorvida pelo trato gastrintestinal, função hepática e renal, capacidade de se ligar a proteínas, uso de medicação 
concomitante que interfere com seu metabolismo, entre outras. O segundo consiste na adição de uma substância inócua ao organismo (ex. vitamina B) que tenha um método de análise mais fácil do que o medicamento em si. É realmente um método indireto, mas que apresenta problemas éticos, uma vez que o paciente deve ser informado de que ingeriu ambas as substâncias ${ }^{(9)}$.

Corroborando com outros estudos que referem sobre os métodos diretos de avaliação e a adesão ao tratamento, o que se observa nesses métodos não apresentam qualquer aspecto prático que os recomende na rotina, por necessitarem de equipamentos sofisticados e de execução difícil e onerosa. A baixa adesão ao regime terapêutico é muito comum, especialmente em doenças crônicas, tornandose um desafio a ser vencido pelos profissionais de saúde ${ }^{(9)}$.

Sobre a terceira e a quarta dimensão de análise, estudos mostraram-se convergentes em vários aspectos de seus resultados. A importância do fornecimento de informação previamente ao controle, tratamento, complicações da hipertensão arterial e os fatores que interferem na não adesão ao tratamento foi reportada pela maioria dos trabalhos. Entretanto, as informações e orientações recebidas nem sempre prepararam suficientemente as pessoas para o controle da doença. Além disso, mesmo quando os pacientes se sentiam bastante informados sobre seu tratamento, ainda havia muitas preocupações e ansiedade com a elevação da pressão arterial e suas complicações. Esse permanece como um grande desafio para os profissionais e o sistema público de saúde.

Os estudos relacionados ao impacto social devido a não adesão ao tratamento da HAS não controlada, considerando a morbidade e mortalidade, é responsável pelo maior ônus social e econômico à população e ao setor saúde. A não adesão ao tratamento é determinada por impactos como: acidente vascular cerebral (AVC), doenças isquêmicas do coração (DAC), insuficiência cardíaca e insuficiência renal. Uma estimativa feita no Brasil, quanto aos hipertensos vinculados ao Sistema Único de Saúde (SUS), revela que os custos hospitalares, entre o período de janeiro a novembro de 2005 , são de $1.932 .751 .952,7$ reais ${ }^{(10)}$.

Moreira e Araújo (2002) concluíram em concordância com outros autores, que os fatores que influenciam na adesão ao tratamento estão relacionados em três categorais: os relacionados diretamente ao paciente, os relacionados à terapêutica e os relacionados ao sistema de saúde ${ }^{(11)}$. 
Em relação à primeira categoria, os fatores que influenciam estão voltados para o sexo do sujeito, idade, grau de escolaridade, condição financeira, estado civil, conhecimentos, crenças, motivação, assiduidade nas consultas, automonitoração da PA, apoio familiar e social que interferem decisivamente na adesão ao tratamento.

De acordo com os estudos, $83 \%$ das mulheres seguiam a dieta hipossódica, sendo que apenas $17 \%$ dos homens seguiam essa recomendação. No estudo realizado por Medel (1997), foram encontrados resultados semelhantes, verificandose que um maior número de mulheres apresentou alto nível de adesão, quando comparados aos homens. Sarquis (1998) demonstrou que as mulheres apresentam uma maior preocupação com a saúde que os homens. Indivíduos de maior idade são mais propensos à adesão. Em pesquisa desenvolvida por Sala et al. (1996), demonstrou-se uma redução de maior intensidade na PAD em pessoas mais velhas. Car et al. (1991) revela que jovens não se sentem vulneráveis à doença, ao passo que o idoso se preocupa mais, no intuito de prolongar a vida através do tratamento ${ }^{(5,8,11)}$.

Verificou-se que o número de indivíduos com curso superior, que se tratava, era seis vezes maior que indivíduos que não tinham ingressado na universidade e realizavam tratamento anti-hipertensivo. Mostrou que 46\% dos hipertensos, com maior nível de escolaridade, estavam dentro do peso normal, enquanto que apenas $24 \%$ dos pacientes com nível educacional mais baixo estavam no peso adequado $^{(5,11)}$.

Sobre a condição financeira, há influência sobre os indivíduos para seguirem recomendações. Segundo Trentini et al. (1996), os pacientes relataram problemas na compra de medicamentos e de alimentos adequados para a dieta do hipertenso. A situação sócioeconômica é um fator altamente relacionado à adesão, não apenas na compra de medicamentos, mas, também, a aspectos culturais, sociais e educacionais $^{(5,11)}$. Hipertensos casados apresentam chance duas vezes maiores de realizar o tratamento, quando comparados com os pacientes solteiros ${ }^{(5)}$.

É de fundamental importância ao hipertenso a adesão ao tratamento, assim como a percepção dos pacientes acerca da doença. A HAS, por ser uma doença muitas vezes assintomática, faz com o que os pacientes não a encarem como um problema de saúde que necessite de tratamento. A ausência de sintomas contribui, de forma marcante, para a não adesão ao tratamento. 
Ressalta-se a dificuldade de convencer o paciente a se tratar, pela ausência de sintomas. A modificação no estilo de vida relaciona-se à presença de sintomatologia, à compreensão dos doentes sobre a doença e ao impacto em suas vidas. A experiência anterior, com algum familiar ou conhecido com algumas complicações da HAS, facilita na adesão ao tratamento. Segundo Marcon et al. (1995), 34,1\% dos hipertensos iniciaram o tratamento por conhecerem pessoas afetadas pelas complicações da $\operatorname{HAS}^{(5,11)}$.

Quanto à terapêutica, medicações que não apresentam resultados, que apresentam efeitos colaterais ou de preço alto, tornam-se obstáculo à adesão ao tratamento. O envolvimento com a terapia constitui um dos fatores que motivam a realização do tratamento. Relata-se a extrema importância do envolvimento do hipertenso em seu autocuidado. Pacientes mais assíduos às consultas tiveram uma maior redução da PA. Consultas freqüentes propiciam uma melhor monitoração dos níveis pressóricos, servindo de base para a adesão. O paciente acompanha a sua doença, o que proporciona uma autorresponsabilidade, com efeito positivo na adesão. Afirmam que é importante a verificação da PA pelo próprio paciente, após treinamento, motivando a adesão(5,11).

A hipertensão arterial provoca alterações no estilo de vida do grupo familiar e não apenas do hipertenso, sendo o apoio familiar um fator facilitador da adesão.

Em relação à terapêutica medicamentosa, a simplificação é um ponto chave. Menor quantidade de medicamentos utilizados, menor número de doses, medicamentos de dose única, diariamente, são fatores que facilitam a adesão. $O$ horário de tomada da medicação é também um fator que intervém na adesão. É necessário relacionar a hora da medicação com as atividades de rotina diária. Alguns autores como Bittar (1995), Pepper (1995), Rudd (1995) e Kjellgren et al. (1995) afirmam que a prescrição medicamentosa deve ser individualizada, elaborada de acordo com o estilo de vida do paciente. Dose única diária, com hora de tomada associada com atividades rotineiras, tem grande importância para a adesão ${ }^{(5,11)}$.

O tratamento, para ser efetivo, deve apresentar pouco ou nenhum efeito colateral na vida do paciente. Medicamentos cada vez mais modernos, com pouco ou nenhum efeito colateral facilitam a adesão do paciente, pois a qualidade de vida melhora. O baixo custo da medicação também é importante na adesão.

Em relação à terapêutica não medicamentosa, há menor taxa de adesão comparada com a terapia medicamentosa, pois ocorrem mudanças problemáticas 
no cotidiano, como: restrições alimentares, lazer e trabalho. Pacientes em uso de medicação têm uma maior dificuldade em aderir à terapia não medicamentosa, pois acreditam que apenas o medicamento é suficiente para o controle da doença ${ }^{(5,11)}$.

Quanto à terceira categoria, o funcionamento do serviço e o relacionamento do paciente com a equipe de saúde influem, fortemente, na adesão ou não adesão do paciente ao tratamento anti-hipertensivo.

A facilidade de entrada do paciente ao serviço de saúde e a qualidade do trabalho desenvolvido são fatores que facilitam a adesão ao tratamento. A disponibilidade de medicamentos também é fator de adesão. Castro e Car (1999) realizaram um estudo com hipertensos e chegaram à seguinte conclusão: medicamentos gratuitos, facilidade de marcar consultas, proximidade do serviço, vale transporte para comparecer às consultas, facilitam a adesão ao tratamento ${ }^{(5,8)}$.

A HAS é uma doença multicausal e multifatorial que exige diferentes formas de abordagem, sendo necessária uma equipe multidisciplinar no atendimento adequado. Uma equipe multidisciplinar é capaz de melhorar, em muito, a adesão terapêutica recomendada. Pode ser constituída de médicos, enfermeiros, nutricionistas, psicólogos, assistentes sociais, professores de educação física, farmacêuticos e funcionários administrativos ${ }^{(5,8)}$.

Outro fator importante na adesão é a freqüência às consultas. Sala et al. (1996) observaram uma redução na PA nas primeiras consultas, cabendo as outras consultas à manutenção da PA já adequada. A satisfação do paciente é apontada como item de grande importância para melhorar o nível de adesão e o controle da doença, sendo necessário um atendimento de boa qualidade. Além de cuidados físicos, os pacientes precisam de estímulo, esperança e compreensão ${ }^{(2,5,8)}$.

\section{Considerações Finais}

A discussão sobre a adesão ao tratamento anti-hipertensivo é de fundamental importância, pois a HAS é uma patologia que, quando não é adequadamente tratada e controlada, gera altos custos financeiros e sociais. O portador de HAS necessita de um tratamento que seja adequado, condizente com a sua situação social, econômica e cultural, e também ter uma medicação associada ao seu estilo de vida. A manutenção de um estilo de vida saudável é um fator que deve ser bastante 
enfatizado pelos profissionais de saúde, devendo ser dadas explicações claras e precisas ao paciente.

A adesão ao tratamento consiste no comportamento que o paciente apresenta diante da sua patologia, do uso da medicação e das modificações no seu estilo de vida que são prescritas pelos profissionais de saúde. A conduta correta do profissional é de suma importância na adesão do paciente ao tratamento, pois este depende de orientações condizentes para o controle adequado da PA. É difícil quantificar a adesão adequada ao tratamento da HAS, mas existem estudos que consideram taxas acima de $80 \%$ como sendo aceitáveis. No Brasil, existem poucos estudos sobre o assunto, mas estima-se que um número entre $40 \%$ e $60 \%$ dos pacientes não fazem uso correto da medicação prescrita. Se forem considerados os pacientes que não modificam o seu estilo de vida, os números são ainda maiores ${ }^{(2)}$.

A HAS não controlada é a doença responsável pelos maiores danos físicos e pelos maiores gastos do sistema de saúde, considerando as taxas de morbidade e mortalidade. A não adesão ao tratamento gera impactos físicos como AVE, doenças isquêmicas cardíacas e insuficiência cardíaca e renal.

O tratamento da HAS é de grande interesse e seu tratamento possui eficácia comprovada. Para tanto, os pacientes devem se esforçar em seguir as prescrições das equipes de saúde, assim como os profissionais de saúde devem adequar-se, prescrevendo a terapêutica adequada ao perfil de cada paciente, a fim de evitar a não adesão ao tratamento e possíveis conseqüências da HAS não tratada.

\section{Referências}

1. SMELTZER, SC; BARE, BG. Brunner \& Suddarth. Tratado de enfermagem médico-cirúrgico. Rio de Janeiro: editora Guanabara Koogan, 2006. p.905.

2. SOCIEDADE BRASILEIRA DE HIPERTENSÃO, SOCIEDADE BRASILEIRA DE CARDIOLOGIA e SOCIEDADE BRASILEIRA DE NEFROLOGIA. V Diretrizes Brasileiras de Hipertensão Arterial, 2006. www.atitude.com.br. Acesso em $11 / 08 / 2008$.

3. MION, JUNIOR D; SILVA, G V; ORTEGA, K C; NOBRE, F. A importância da medicação anti-hipertensiva na adesão ao tratamento. Revista Brasileira de Hipertensão. v.13,n.1, 2006. p.55-58. 
4. JARDIM, P C B V. Modelos de estudos de adesão ao tratamento anti-hipertensivo. Revista Brasileira de Hipertensão. v.13,n.1 2006. p.26-29.

5. ARAÚJO, G B S.; GARCIA, T R. Adesão ao tratamento anti-hipertensivo: uma analise conceitual. Revista Eletrônica de Enfermagem, v.08,n.02, 2006. p.259-272. Disponível em http://www.fen.ufg.br/revista/revista8 2/v8n2a11.htm

6. BARBOSA, R G B; LIMA, N K C. Índices de adesão ao tratamento antihipertensivo no Brasil e mundo. Revista Brasileira de Hipertensão v.13,n.1, 2006. p.35-38.

7. GUSMÃO, J L; MION JUNIOR, D. Adesão ao tratamento - conceitos. Revista Brasileira de Hipertensão. v.13,n.1, 2006. p.23-25.

8. SANTOS, Z M S A; FROTA, M A; CRUZ, D M; HOLANDA, S D O. Adesão do cliente hipertenso ao tratamento: análise com abordagem interdisciplinar. Texto Contexto Enferm.; v.14,n.3, Jul./Set., 2005. p.332-340.

9. OIGMAN, W. Métodos de avaliação da adesão ao tratamento anti-hipertensivo. Revista Brasileira de Hipertensão. v.13,n.1, 2006. p.30-34.

10. LESSA, I. Impacto social da não adesão ao tratamento da hipertensão arterial. Revista Brasileira de Hipertensão. v.13,n.1. 2006. p.39-46.

11. MOREIRA, T M M; ARAÚJO, T L. Sistema interpessoal de Imogene King: as relações entre pacientes com hipertensão não aderentes ao tratamento e profissionais de saúde. Acta Paul. Enf. v.15,n.3, 2002. p.35-42. 